\title{
Surgical management of urothelial cancer in a cirrhotic patient after partial splenic embolisation
}

\author{
Ion Dina ${ }^{1,2}$, Cornelia Voiculet ${ }^{1,2}$, Dragoș Georgescu ${ }^{3,4}$, Adriana Luminița Gurghean ${ }^{2,5}$, Alina Pleșa ${ }^{6,7}$, \\ Octavian Dumitru Zară ${ }^{1}$ \\ 1Department of Internal Medicine, “St. John” Emergency Clinical Hospital Bucharest, Bucharest, Romania \\ ${ }^{2}$ Clinical Department No. 1, "Carol Davila" University of Medicine and Pharmacy Bucharest, Bucharest, Romania \\ ${ }^{3}$ Department of Urology, "St. John” Emergency Clinical Hospital Bucharest, Bucharest, Romania \\ ${ }^{4}$ Clinical Department No. 3, "Carol Davila" University of Medicine and Pharmacy Bucharest, Bucharest, Romania \\ ${ }^{5}$ Department of Internal Medicine, "Colțea” Hospital Bucharest, Bucharest, Romania \\ ${ }^{6}$ Department of Gastroenterology, "St. Spiridon” Emergency Clinical Hospital Iași, Iași, Romania \\ ${ }^{7}$ Clinical Department, “Grigore T. Popa” University of Medicine and Pharmacy lași, Iași, Romania
}

Gastroenterology Rev 2019; 14 (4): 298-301

DOI: https://doi.org/10.5114/pg.2019.90256

\begin{abstract}
Address for correspondence: Cornelia Voiculet MD, PhD, Department of Internal Medicine "St. John" Emergency Clinical Hospital Bucharest,
\end{abstract} $13^{\text {th }}$ Vitan-Bârzeşti, Bucharest, Romania, e-mail: cornelia_voiculet@yahoo.com

A 50-year-old male patient with known liver cirrhosis due to chronic hepatitis $B$ virus infection was referred to our service for partial splenic embolisation. The patient was diagnosed 1 month earlier with urothelial cancer. The biological assessment revealed severe thrombocytopaenia of 44,000 platelets $/ \mathrm{mm}^{3}$. The past medical history showed only viral liver cirrhosis in a compensated state. Given the significant bleeding risk, the multidisciplinary team decided that the patient was not a candidate for surgical nephrectomy, and partial splenic embolisation was proposed as a minimally invasive procedure in an attempt to restore the peripheral blood cell count back to normal. At admission to the gastroenterology department the patient had 50,000 platelets $/ \mathrm{mm}^{3}$ and the creatinine level was $1.13 \mathrm{mg} \%$ - modification of diet in renal disease $(M D R D)=76 \mathrm{ml} / \mathrm{min} / 1.73 \mathrm{~m}^{2}$. Upper digestive endoscopy revealed oesophageal varices of grade I-II. Computed tomography scan of the abdomen showed marked splenomegaly in addition to cirrhosis-like hepatomegaly, portal hypertension, minimal pelvic ascites, and urothelial left kidney tumour (Figure 1).

The patient gave consent to perform the procedure after being informed of its risks and benefits. The right common femoral artery was accessed, and the initial abdominal aortogram showed the origin of the celiac trunk (Figure 2). Then the splenic artery was catheterised using a 6 Fr catheter (Boston Scientific), and a splenic angiogram showed the spleen vascularisation (Figure 3). The catheter was then advanced through the lower polar splenic artery. Angiography confirmed the location in the distal splenic artery (Figure 4). Partial embolisation of the lower third of the spleen was achieved using haemostatic sponge (Sponjel). Follow-up angiogram showed complete stasis in the embolised segments of the spleen (Figure 5).

Continued follow-up of the patient over a period of 14 days showed gradual improvement of peripheral blood cell count, which returned back to normal levels (platelet count from 44,000 to 156,000 platelets $/ \mathrm{mm}^{3}$ ) (Figure 6). Follow-up abdominal ultrasound performed 2 days after the procedure revealed a hypodense area localised at the inferior spleen pole, corresponding to the splenic infarction that resulted from the previous embolisation and also a hypoechogenic area in the left kidney. The patient was transferred to the urology department 14 days after the procedure, in order to determine the opportunity for nephrectomy. Considering the extent of surgery and the related pathologies, it was decided to perform splenectomy, in addition to nephrectomy. Thus, 2 weeks after the partial splenic embolisation, when the platelet count returned to normal but the creatinine level increased to $1.69 \mathrm{mg} \%$ (MDRD = $47 \mathrm{ml} / \mathrm{min} / 1.73 \mathrm{~m}^{2}$ ), surgical resection of the renal tumour together with splenectomy were performed (Figure 7). The histopathological examination of the renal formation revealed urothelial carcinoma (Figure 8).

The patient had a favourable outcome and monitoring of bioumoral assays over the next 7 days revealed an increase in platelet counts up to $399,000 / \mathrm{mm}^{3}$ 


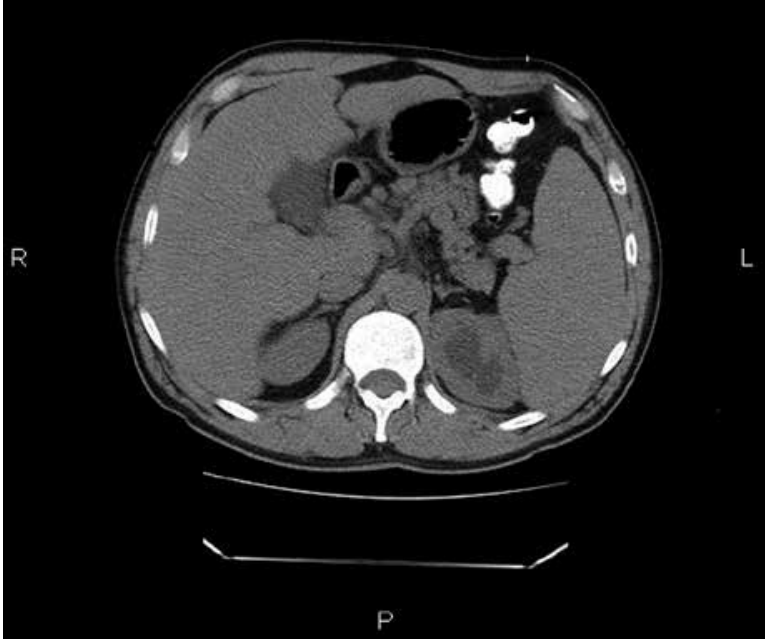

Figure 1. Urothelial left kidney tumour

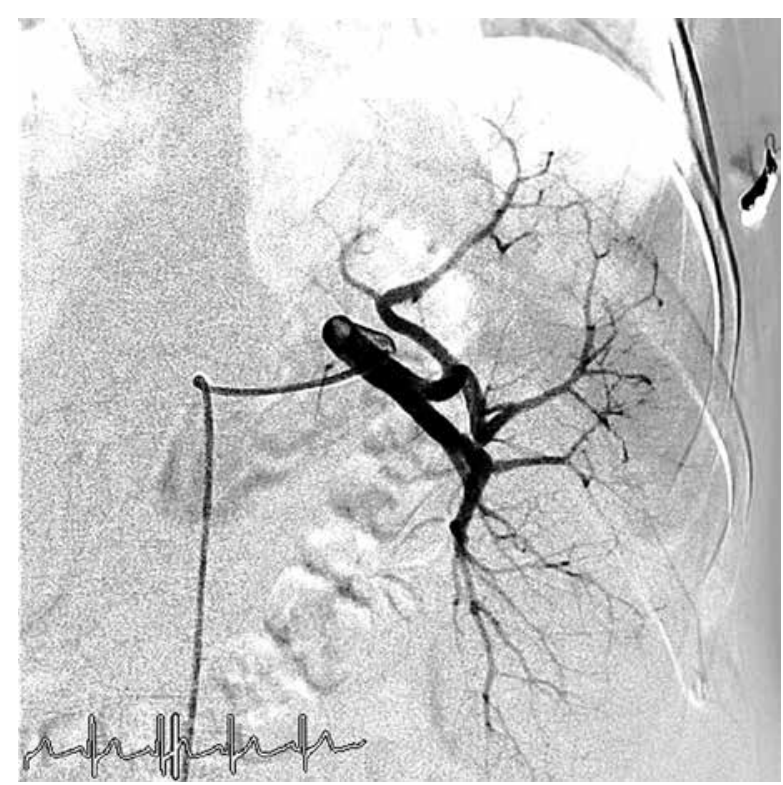

Figure 3. Spleen vascularisation

(Figure 9) and creatinine level recovery within normal range to $1.17 \mathrm{mg} \%$ (MDRD $=70 \mathrm{ml} / \mathrm{min} / 1.73 \mathrm{~m}^{2}$ ). The patient was discharged with the recommendation to perform periodic gastroenterological control to monitor oesophageal varices, periodic urological control, oncological follow-up, and anti-pneumococcal, anti-meningococcal, anti-haemophilus influenzae vaccination.

Short-term prognosis was favourable in terms of normal platelet restoration (following splenectomy) and ureteral tumour excision, as opposed to long-term prognosis, which was critical due to increased risk of septic events, distant metastasis development, and complications of liver cirrhosis. The particularity of the case consists of potential surgical haemorrhagic risk

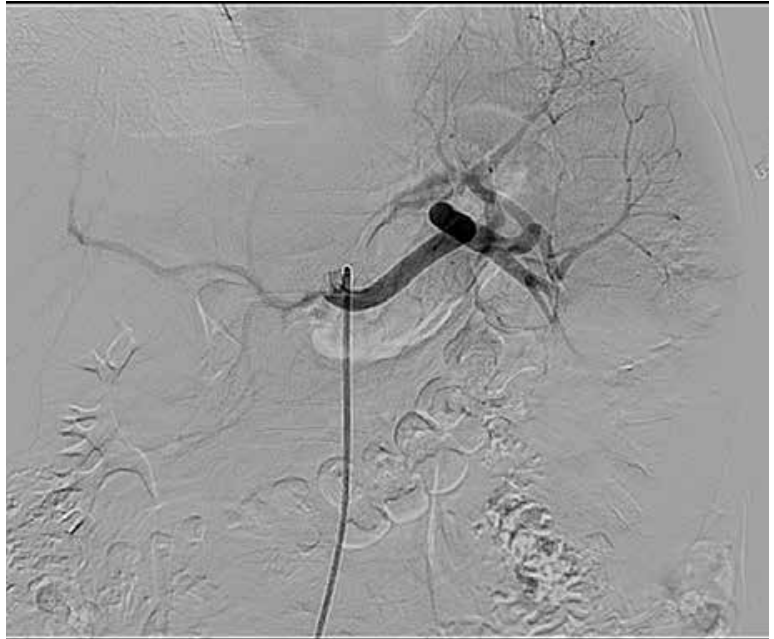

Figure 2. The origin of the celiac trunk

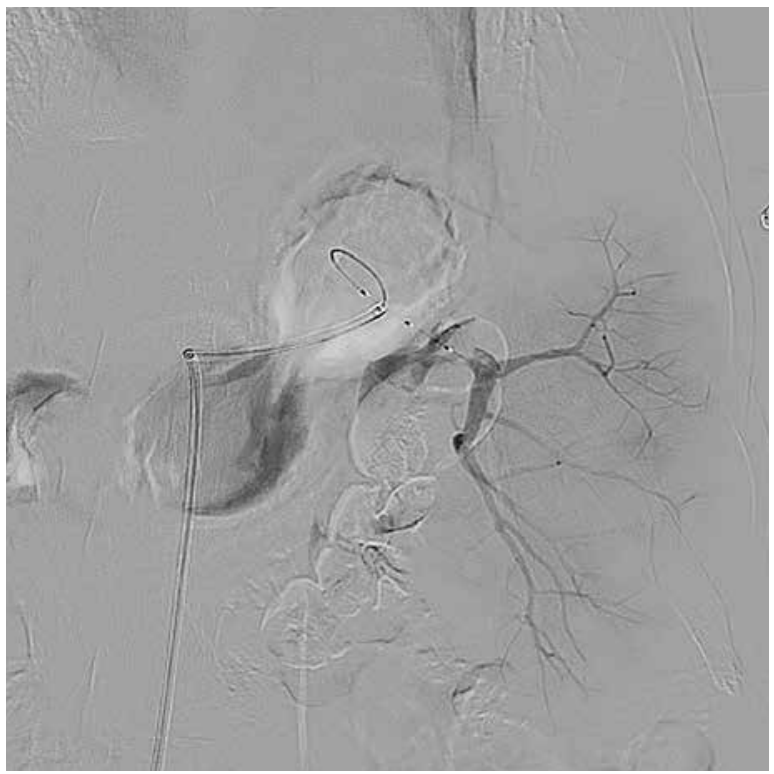

Figure 4. The lower polar splenic artery

due to severe thrombocytopaenia in a young patient with hepatic cirrhosis and preserved liver function, who required nephrectomy for urothelial cancer.

Portal hypertension is a common consequence of liver cirrhosis and frequently leads to splenomegaly, which is often associated with a decrease in haematological indices including thrombocytopaenia. Several treatment options, including platelet transfusion, surgical splenectomy, and interventional splenic artery embolisation, are now available for thrombocytopaenia in patients with liver cirrhosis [1, 2]. Partial splenic embolisation was demonstrated to effectively increase haematological indices in cirrhotic patients with hypersplenism for the treatment of hypersplenism. Maddison 


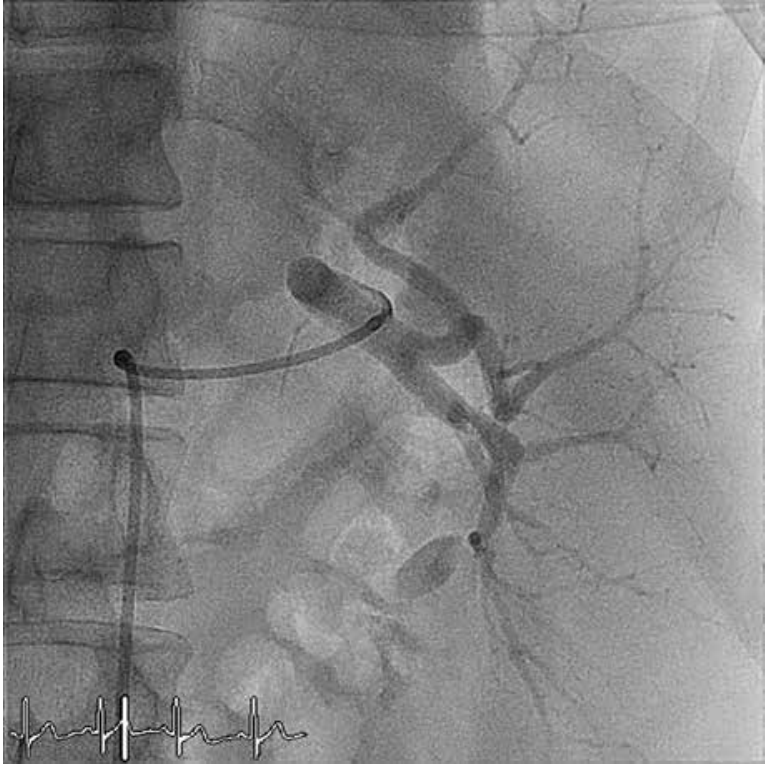

Figure 5. The embolised segments of the spleen

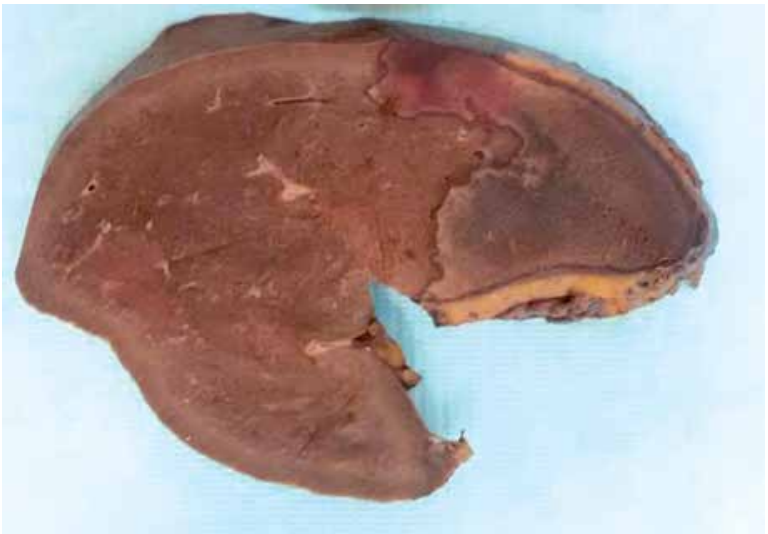

Figure 7. The anatomopathological examination of the spleen

proposed in 1973 an effective technique: splenic artery embolisation as a minimally invasive procedure. Despite Maddison's early success, numerous complications of total splenic artery embolisation were discovered: splenic rupture, splenic abscess, septicaemia, pneumonia, and death. Soon partial splenic embolisation gained popularity and served as a therapeutic option for cirrhotic patients with hypersplenism requiring surgery for various pathologies [1, 3-8]. Decreased haematological indices have severe clinical consequences. Thrombocytopaenia increases the risk of spontaneous bleeding in patients and may preclude surgical or endovascular interventions [1, 2]. Considering the data from the literature and the clinical and paraclinical state of our patient (urothelial cancer, cirrhosis with oesophageal varices and hypersplenism - severe thrombocytopaenia), the risk of bleeding was reduced by performing partial em-

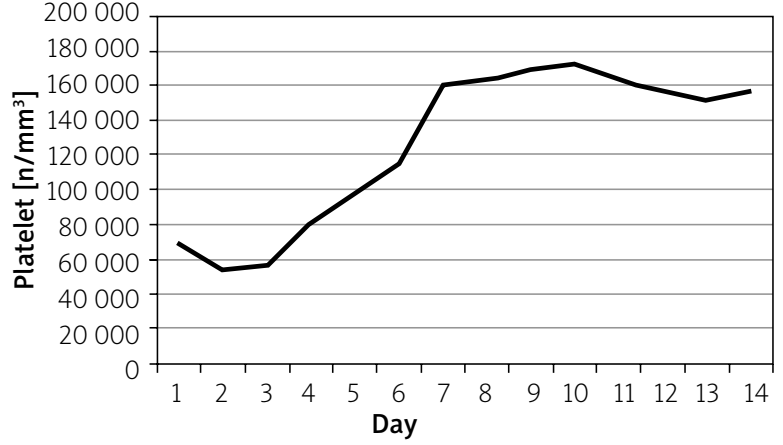

Figure 6. The evolution of platelet count after partial splenic embolisation

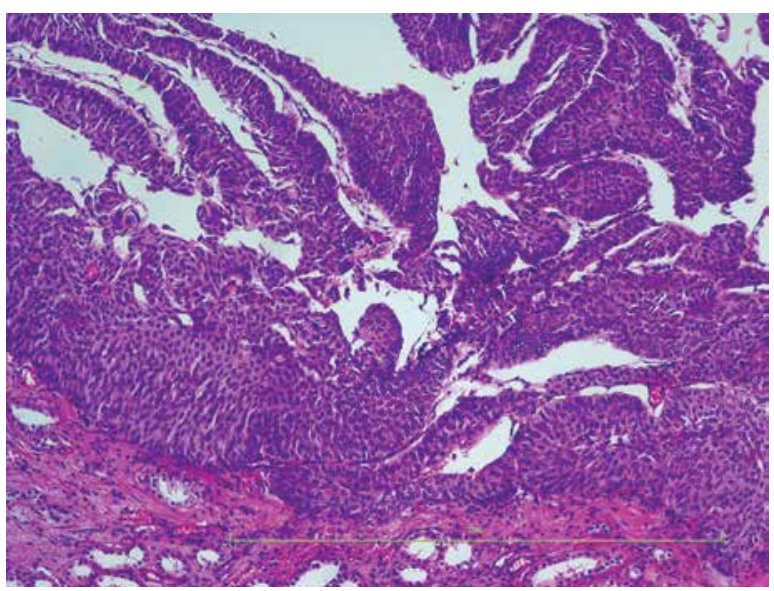

Figure 8. Histopathological examination of the renal tumour

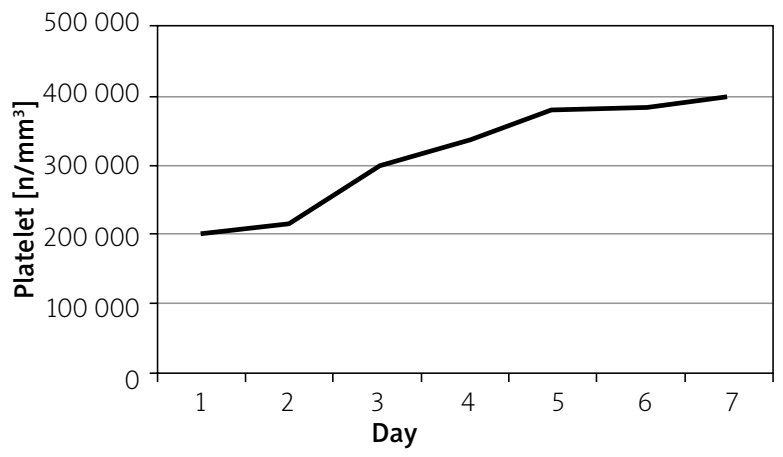

Figure 9. The evolution of platelet count after splenectomy

bolisation of the spleen in order to increase the number of platelets. The platelet number returned to normal $\left(160,000\right.$ platelet $\left./ \mathrm{mm}^{3}\right) 7$ days after partial splenic embolisation and remained within normal range, and after 2 weeks the patient could benefit from nephrectomy $[1,2]$. Clinical studies have shown that embolisation of less than $50 \%$ of the spleen has been associated with shorter hypersplenism relapses, suggesting that partial 
splenic embolisation should cover a minimum of $50 \%$ of the spleen to be effective $[9,10]$.

The data from the literature highlights that the longterm efficacy of partial splenic embolisation and improvement in cytopenia is directly correlated with the rate of splenic infarction. We found that platelet counts increased post partial splenic embolisation, reaching a peak at day 10 , followed by a short period of gradual decrease after surgery, and then by a spectacular increase after urological intervention and spleen removal. The patient will be periodically evaluated by a gastroenterologist to monitor the liver function, oesophageal varices, and platelet count. The urologist and the nephrologist will monitor renal function, and the oncologist will administer treatment for urothelial cancer according to the histopathological exam. The secondary infectious risk of splenectomy will be controlled by regular vaccination.

The interdisciplinary therapeutic approach of the patient with urothelial cancer and severe thrombocytopaenia was successful for radical urologic surgery as well as for the decrease of the haemorrhagic risk due to severe thrombocytopaenia through partial splenic embolisation.

\section{Conflict of interest}

The authors declare no conflict of interest.

\section{References}

1. Hadduck TA, McWilliams JP. Partial splenic artery embolization in cirrhotic patients. World J Radiol 2014; 6: 160-8.

2. Hiromitsu H, Toru B, Ken S, et al. Management of thrombocytopenia due to liver cirrhosis: a review. World J Gastroenterol 2014; 20: 2595-605.

3. Souheil S, Amr SM, Hany AT, et al. Treatment of hypersplenism by partial splenic embolization trough gastriccollaterals. Intervent Radiol 2016; 10: 28-35.

4. Spigos DG, Jonasson O, Mozes M, Capek V. Partial splenic embolization in the treatment of hypersplenism. AJR Am J Roentgenol 1979; 132: 777-82.

5. Dwivedi MK, Pal PK, Dewanga L, Nag P. Efficacy of partial splenic embolisation in the management of hypersplenism. Indian J Radiol Imaging 2002; 12: 371-4.

6. Hamblin TJ, Oscier DG, Young BJ. Autoimmunity in chronic lymphocytic leukaemia. J Clin Pathol 1986; 39: 713-6.

7. Maddison FE. Embolic therapy of hypersplenism. Invest Radiol 1973; 8: 280-300.

8. Castaneda-Zuniga WR, Hammerschmidt DE, Sanchez R, Amplatz K. Nonsurgical splenectomy. AJR Am J Roentgenol 1977; 129: 805-11.

9. Omer O, Gonca E, Bora P, et al. Outcomes of partial splenic embolization in patients with massive splenomegaly due to idiopathic portal hypertension. World J Gastroenterol 2016; 22: 9623-30.

10. Petermann A, Chabrot P, Cassagnes L, et al. Hypersplenism due to portal hypertension: retrospective evaluation of 17 patients treated by splenic embolization. Diagn Interv Imaging 2012; 93: 30-6.

Received: 19.03.2019

Accepted: 18.04 .2019 